\title{
Augmenter of Liver Regeneration in ROS- induced Pathophysiological Conditions
}

\author{
Sudhir Kumar* and Richa Rani \\ Department of Pediatrics, Cincinnati Children's Hospital Medical Center, USA
}

*Corresponding author: Sudhir Kumar, Division of Gastroenterology, Hepatology and Nutrition, Department of Pediatrics, Cincinnati Children's Hospital Medical Center, Cincinnati, Ohio, USA, Tel: +1-513-517-0584; Email: sudhir.kumar@cchmc.org

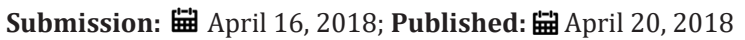

Keywords: Antioxidant; Augmenter of liver regeneration; Liver injury; Oxidative stress; Reactive oxygen species:

\section{Editorial}

Augmenter of liver regeneration (ALR), also described as hepatopoietin (HPO) and hepatic stimulator substance or hepatic regenerative stimulation substance(HSS), is representative member of ERV protein family with flavin-linked sulfhydryl oxidase activity, that facilitate the formation of disulfide bonds in reduced protein substrates [1]. Growing numbers of evidence demonstrate the involvement of ALR in maintaining cellular redox communication under various pathophysiological conditions [2-7]. Understanding redox regulation and precise assessment of metabolic/oxidative stress are key to monitor diverse range of pathophysiological events, since a large number of cellular functions and dysfunctions are, either directly or indirectly, triggered in response to redox signals. Reactive oxygen species (ROS) which are highly reactive molecules can be generated within a cell in response to different environmental stimuli (growth factors, inflammatory cytokines, ionizing radiation, UV, chemical oxidants, chemotherapeutics, hyperoxia, toxins, and transition metals), by-products of aerobic respiration, catabolic and anabolic processes and activated immune cells. Hepatotoxic agents are the greatest risk factor for oxidative stress-induced diseases and there is strong evidence that they trigger formation of intracellular ROS and accumulation of radicals contributing to both inflammation and cell death. Antioxidant proteins and enzymes such as, catalase, glutathione peroxidase, and thioredoxin peroxidase, responsible for detoxifying the electrophiles and radicals, have long been established as the crucial players in the defense of oxidative stress. In liver disease, oxidative stress in general has been reported as a resultant phenomenon of decreased antioxidant defense, increased release of oxidant enzymes, generation of ROS, neutrophil dysfunction, and enhanced oxidative modifications on lipids. Studies have revealed alterations in cellular membrane integrity, oxidative deterioration of DNA, protein, and lipid as the fundamental detrimental effects of ROS (superoxide anion (O2-), hydroxyl radical $(\bullet \mathrm{OH})$, and hydrogen peroxide (H2O2), ultimately leading to dysfunction of cellular compartments. Mitochondria are considered as the primary target of oxidative damage and at the same time the major source of intracellular ROS, resulting into etiopathogenesis of hepatotoxicity (oxidative stress, lipid peroxidation and impaired homeostasis of ions and glutathione) and might play an important role in pathophysiology of liver diseases. Many lines of evidence suggest that mitochondrial dysfunction affects energy balance and lipid metabolism in the hepatocytes and acts causally in disease pathogenesis [5,7]. The mitochondrial function of ALR is apparent from their localization in the inter-membrane space of the organelle [8] and in this context, the functions of ALR have been elucidated by many researchers, but we still lack the knowledge of precise correlation between ROS-induced oxidative damage, mitochondrial dysfunction, and hepatoprotective role of ALR as an antioxidant. In this context, Gandhi et al. [5] have investigated the precise role of this protein in hepatic physiology and pathology, as well as, on how ALR impact cellular physiology during oxidative stress. Additionally, deficiency of ALR in liver is associated with exacerbation of stressor-induced liver diseases. Our recent report indicates that under ALR-deficient condition, ethanol feeding to mice strongly augments liver injury and affects ethanol metabolism possibly due to low ALDH activity [7]. Another study by Shi et al. [9] revealed that ALR protects from chemical-induced liver damage by enhancing autophagic mechanism. Despite several new findings, their implementation in prevention, reversal, or delaying the onset of human diseases is still lacking. There is a need to make research more relevant, to fill the translational gap between animaland human-based studies, by identifying common molecular mechanisms and their effective targets.

\section{References}

1. Thorpe C, Coppock DL (2007) Generating disulfides in multicellular organisms: emerging roles for a new flavoprotein family. J Biol Chem 282(19): 13929-13933. 
2. Polimeno L, Pesetti B, Annoscia E, Giorgio F, Francavilla R, et al. (2011) Alrp, a survival factor that controls the apoptotic process of regenerating liver after partial hepatectomy in rats. Free Radic Res 45(5): 534-549.

3. Polimeno L, Pesetti B, De Santis F, Resta L, Rossi R, et al. (2012) Decreased expression of the augmenter of liver regeneration results in increased apoptosis and oxidative damage in human-derived glioma cells. Cell Death Dis 3: e289.

4. Polimeno L, Rossi R, Mastrodonato M, Montagnani M, Piscitelli D, et al (2013) Augmenter of liver regeneration, a protective factor against ROSinduced oxidative damage in muscle tissue of mitochondrial myopathy affected patients. Int J Biochem Cell Biol 45(11): 2410-2419.

5. Gandhi CR, Chaillet JR, Nalesnik MA, Kumar S, Dangi A, et al. (2015) Liver-specific deletion of augmenter of liver regeneration accelerates development of steatohepatitis and hepatocellular carcinoma in mice. Gastroenterology 148(2): 379-391.
6. Xia N, Yan RY, Liu Q, Liao XH, Sun H, et al. (2015) Augmenter of liver regeneration plays a protective role against hydrogen peroxide-induced oxidative stress in renal proximal tubule cells. Apoptosis 20(4): 423-432.

7. Kumar S, Wang J, Rani R, Gandhi CR (2016) Hepatic deficiency of augmenter of liver regeneration exacerbates alcohol-induced liver injury and promotes fibrosis in mice. PLoS One 11(1): e0147864.

8. Lange H, Lisowsky T, Gerber J, Mühlenhoff U, Kispal G, et al. (2001) An essential function of the mitochondrial sulfhydryl oxidase Erv1p/ALR in the maturation of cytosolic Fe/S proteins. EMBO Rep 2(8): 715-720.

9. Shi H, Han W, Shi H, Ren F, Chen D, et al. (2017) Augmenter of liver regeneration protects against carbon tetrachloride-induced liver injury by promoting autophagy in mice. Oncotarget 8(8): 12637-12648.
Creative Commons Attribution 4.0

International License

For possible submissions Click Here
Submit Article

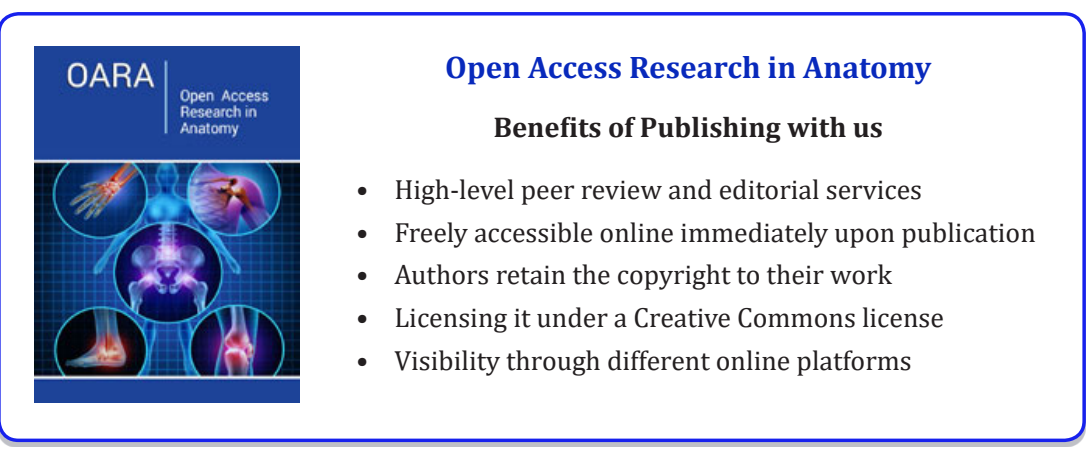

